\title{
Regulation of TLR2 and NLRP3 in Primary Murine Renal Tubular Epithelial Cells
}

\author{
Sashi G. Kasimsetty Sean E. DeWolf Alana A. Shigeoka Dianne B. McKay \\ Division of Nephrology-Hypertension, Department of Medicine, University of California San Diego, \\ San Diego, Calif., USA
}

\section{Key Words}

Renal tubular epithelial cells · Pattern recognition

receptors $\cdot$ Regulation

\begin{abstract}
Pattern recognition receptors (PRRs) are now recognized to be key triggers of injury in a variety of renal diseases. Several families of these receptors are present in the kidney, and recent data suggest that they are differentially expressed and regulated in the kidney. This study evaluated the interaction between two distinct PRRs that are expressed in the kidney, i.e. TLR2 (Toll-like receptor 2 ) and the NLRP3 inflammasome. The regulation and activation of these receptors in primary renal tubular epithelial (RTE) cells from murine kidneys were evaluated. RTE cells were extracted from WT and NLRP3-mutant mice and treated ex vivo with ligands specific for TLR2 or NLRP3. We found that TLR2 upregulated NLRP3 as well as its substrate IL-1 $\beta$, and that signaling through the NLRP3 inflammasome induced RTE cell necrosis. The results of this study suggest a previously unknown interaction between TLR2 and NLRP3 in primary RTE cells and highlight the importance of the cross talk that occurs in kidney-related PRRs. Understanding how PRRs are regulated is important for the design of rationale therapeutic strategies to modulate these receptors in renal disease.

(c) 2014 S. Karger AG, Basel
\end{abstract}

\section{Introduction}

Innate immune receptors [also called pattern recognition receptors (PRRs)] are taking center stage as essential triggers of acute and chronic renal injury. These receptors are known to induce proinflammatory signals and death signaling in response to a wide variety of pathogen and nonpathogen stimuli. The complexity of PRR signaling is only now being appreciated in the kidney.

Emerging studies have shown that significant cross talk exists between PRR family members and that differential expression of PRRs occurs throughout the kidney. Our study was designed to clarify the interaction between two different PRRs known to be expressed in both rodent and human kidneys, i.e. TLR2 (Toll-like receptor 2) and the NLRP3 (nucleotide-binding oligomerization domain-like receptor family, pyrin domain containing 3 , also known as NALP3 and cyropyrin) inflammasome.

Changing Paradigms in Acute Kidney Injury: From Mechanisms to Management - Proceedings of the 5th Annual UAB-UCSD O'Brien Center Symposium (San Diego, Calif., USA, March 4, 2014).

S.G. Kasimsetty and S.E. DeWolf contributed equally to this work.

\section{KARGER}

E-Mail karger@karger.com

www.karger.com/nec
(C) 2014 S. Karger AG, Basel

$1660-2110 / 14 / 1274-0119 \$ 39.50 / 0$
Dr. Dianne McKay

Division of Nephrology-Hypertension, Department of Medicine University of California San Diego 9500 Gilman Drive, San Diego, CA 92093 (USA)

E-Maild1mckay@ucsd.edu 
TLR2 is a membrane-bound PRR that responds to cell wall components such as peptidoglycan, lipoteichoic acid, and lipoprotein, depending on the coreceptors that interact with it. TLR2 is expressed in proximal tubular epithelium and its blockade prevents renal ischemia/reperfusion injury in rodent models [1]. The NLRP3 inflammasome is a multiprotein complex that is formed in response to a wide variety of infectious and noninfectious stress signals. Formation of the inflammasome complex promotes autocatalytic cleavage of caspase 1 , which then enzymatically cleaves precursor pyrogens such as IL-1 $\beta$ into their mature secreted form and induces caspase-1-dependent cell death (pyroptosis). TLR2 has been shown to be an upstream signal for NLRP3 inflammasome activation in several in vitro studies in inflammatory cells [2]. During a stress response two signals have been shown to be required for inflammasome complex formation and subsequent caspase- 1 activation. The first ensures adequate gene/protein expression of pro-IL- $1 \beta$ and inflammasome components (e.g. NLRP3), while a second signal is required for inflammasome complex assembly and subsequent caspase-1 activation.

Our study investigated whether signaling through TLR2 influences activation of the NLRP3 inflammasome in primary renal tubular epithelial (RTE) cells and whether activation of the NLRP3 inflammasome leads to death of the RTE cells. These studies set the stage to better define potential synergistic interactions between families of PRRs in the kidney. Understanding the cooperative communication between PRR family members is essential for the design of rationale therapeutics to prevent injurious responses in the kidney.

\section{Methods}

\section{Isolation of Primary RTE Cells}

RTE cells were extracted from adult male mice (aged 6-9 weeks) using a previously published methodology [1, 3-5]. WT (C57Bl/6) mice were compared to NLRP3-/- mice (bred for more than 10 generations on a $57 \mathrm{Bl} / 6$ background, a gift from $\mathrm{H}$. Hoffman of the University of California San Diego). Mice were sacrificed and kidneys were immediately excised and placed in ice-cold complete media (10\% heat-inactivated FBS; Omega Scientific, Tarzana, Calif., USA) in RPMI 1640 supplemented with 1\% penicillin-streptomycin (Life Technologies, Grand Island, N.Y., USA). The renal capsule was removed, the kidneys were cut sagittally, and the medulla was discarded. The cortical tissue was minced in collagenase solution in complete media $(1 \mathrm{mg} / \mathrm{ml}$; Sigma-Aldrich, St. Louis, Mo., USA). The tubules were incubated at $37^{\circ} \mathrm{C}$ for $45 \mathrm{~min}$ with frequent mixing. The tubule fragments were sieved $(100 \mu \mathrm{m})$ and washed twice with complete media followed by centrifugation in a $30 \%$ Percoll gradient at $12,500 \mathrm{rpm}$ for 30 min at $4^{\circ} \mathrm{C}$. Proximal tubule fragments and cells were retrieved from the layer directly above the red blood cells and washed twice with RPMI and centrifuged at $1,200 \mathrm{rpm}$ for $5 \mathrm{~min}$ at $4^{\circ} \mathrm{C}$. Proximal tubule epithelial cells were seeded on collagen-treated plates (BD Biosciences, San Jose, Calif., USA) using selection media consisting of DMEM:F12 culture media (Life Technologies), 1\% penicillin-streptomycin (Life Technologies), hydrocortisone (50 nM; Sigma-Aldrich), epidermal growth factor (10 ng/ml; Sigma-Aldrich), insulin/transferrin/selenium $(10 \mu \mathrm{g} / \mathrm{ml} / 5.5 \mu \mathrm{g} / \mathrm{ml} / 5 \mathrm{ng} / \mathrm{ml}$; Sigma-Aldrich), and tri-iodothyronine (32 ng/ml; Sigma-Aldrich), placed in an incubator $\left(37^{\circ} \mathrm{C}, 5 \% \mathrm{CO}_{2}\right)$, and grown to $80 \%$ confluence.

\section{Detection of NLRP3 and IL-1 $\beta$ Message}

To determine the relative expression of NLRP 3 or IL- $1 \beta$ mRNA following stimulation, the primary RTE cells were serum-starved overnight in DMEM:F12 (Life Technologies), followed by treatment with either Pam3Cys-Ser-(Lys) 4 trihydrochloride (specific to TLR1/2) (100 ng/ml), also called PAM3 (100 ng/ml; Enzo Life Sciences, Farmingdale, N.Y., USA), or ATP (3 mM; Sigma-Aldrich). To allow for ligand binding, the cells were then exposed to $4^{\circ} \mathrm{C}$ for $15 \mathrm{~min}$, followed by stimulation at $37^{\circ} \mathrm{C}$ with $5 \% \mathrm{CO}_{2}$ for $4 \mathrm{~h}$. After $4 \mathrm{~h}$, the medium was removed, the cells were washed with sterile PBS, and adherent cells were scraped and pelleted. The pellets were washed with sterile PBS and stored at $-80^{\circ} \mathrm{F}$ for detection of mRNA.

RNA was purified using a Quick-RNA MiniPrep kit (Zymo Research Corp., Irvine, Calif., USA). The first strand of cDNA of each sample was synthesized from 500 ng total RNA using a SuperScript III Reverse Transcriptase kit (Life Technologies) according to the manufacturer's instructions. qPCR was run and quantified using an Eco Real-Time PCR System (Illumina, San Diego, Calif., USA) detecting the amplification of SYBR Green fluorescent signals using SsoAdvanced SYBR Green Supermix (Bio-Rad, Hercules, Calif., USA) and primer sets for IL-1 $\beta$ and NLRP3 (SA Biosciences, Valencia, Calif., USA).

\section{NLRP3-Induced RTE Cell Death}

To assess for ligand-induced cell death, primary RTE cells from either WT or NLRP3-/- mice were stimulated with either PAM3 $(10 \mu \mathrm{g} / \mathrm{ml})$ or ATP $(3 \mathrm{mM})$ or nigericin $(5 \mu \mathrm{g} / \mathrm{ml})$ for $24 \mathrm{~h}$. After stimulation, the cells were harvested and suspended in annexin $\mathrm{V}$ binding buffer (10 mM Hepes, $0.14 \mathrm{M} \mathrm{NaCl}$, and $2.5 \mathrm{mM} \mathrm{CaCl}_{2}$ solution; BD Biosciences) and then stained with annexin $\mathrm{V}$ (Nexcelom Bioscience, Lawrence, Mass., USA) and propidium iodide (PI; Nexcelom Bioscience) for $15 \mathrm{~min}$ at room temperature. The cells were then washed with annexin $\mathrm{V}$ binding buffer and analyzed for annexin V/PI uptake using a Nexcelom cellometer and FCS4 Express software (Nexcelom Bioscience).

To detect HMGB1 nuclear release, RTE cells were treated with either PAM3 $(10 \mu \mathrm{g} / \mathrm{ml})$ or ATP $(5 \mathrm{mM})$ for $24 \mathrm{~h}$. After stimulation, $2 \mathrm{ml}$ supernatant was added to double the volume of cold acetone and stored at $-20^{\circ} \mathrm{C}$ overnight to precipitate the protein. The protein precipitate was collected by spinning the supernatant at $17,000 \mathrm{~g}$ for $10 \mathrm{~min}$. The lysis buffer (60 mM Tris, $10 \%$ glycerol, $2 \%$ SDS and protease inhibitors) was added to the collected pellet to solubilize the protein. The concentration of soluble protein was determined by Lowry assay (Bio-Rad), and $30 \mu \mathrm{g}$ protein was loaded and resolved on a $12 \%$ electrophoresis gel followed by PVDF 


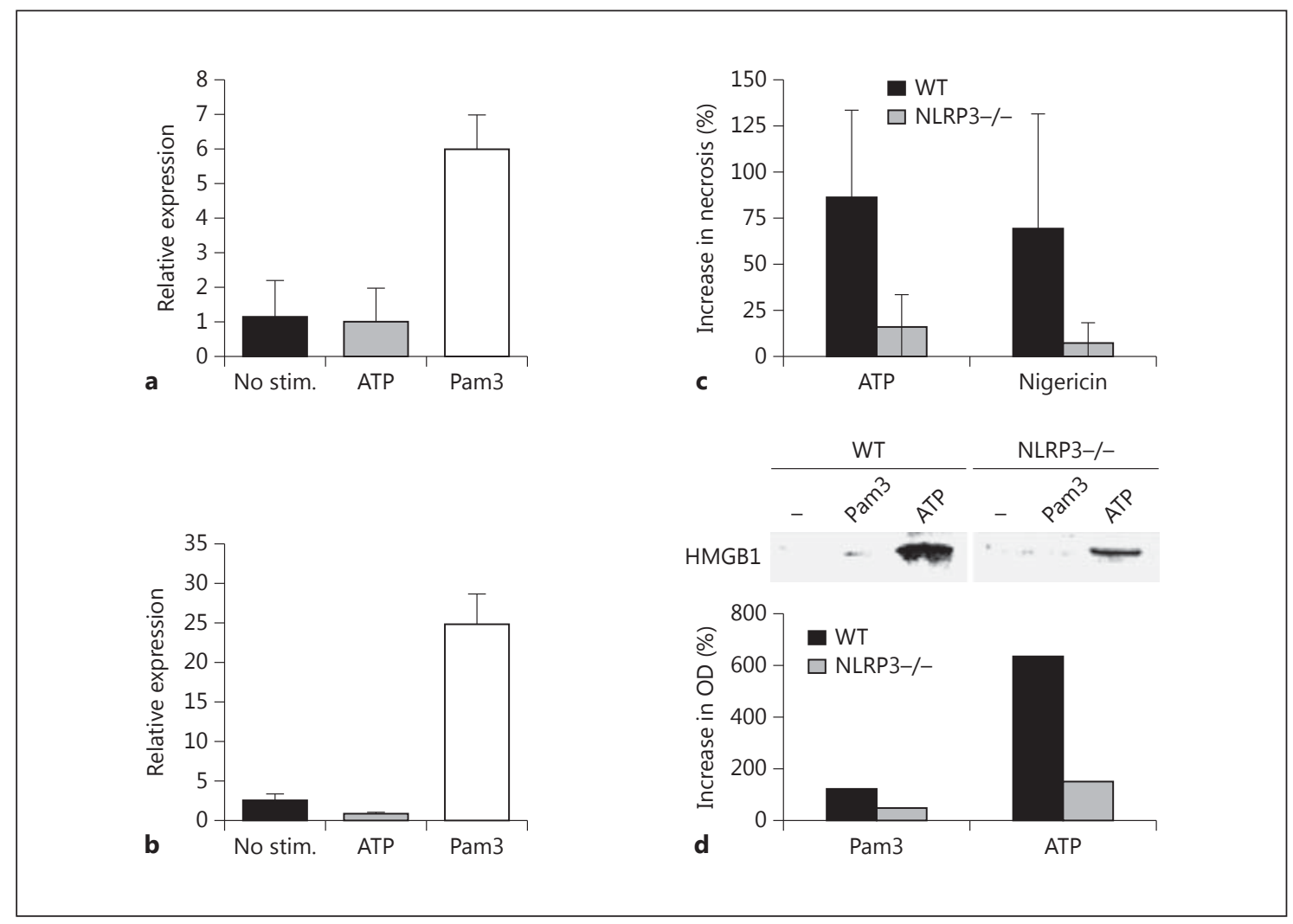

Fig. 1. Upregulation of NLRP3 and IL- $1 \beta$ and ATP-induced necrosis in primary RTE cells. a Induction of NLRP 3 mRNA in primary RTE cells from WT (C57Bl/6) mice. RTE cells were either not stimulated (No stim.) or exposed to ATP or Pam 3 for 4 h. b Induction of IL-1 $\beta$ mRNA in primary RTE cells from WT mice without stimulation (No stim.) or after $4 \mathrm{~h}$ of ATP or Pam 3 exposure. a, b The error bars represent the standard deviations of 2 replicates and are representative of 4 similar experiments. c Ligand-induced RTE cell death in WT (C57Bl/6) versus NLRP3-/- mice. The bars represent the percent

membrane transfer and Western blot for HMGB1 protein $(29 \mathrm{kDa})$ using a polyclonal anti-HMGB1 rabbit polyclonal antibody (Abcam, Cambridge, Mass., USA).

\section{Results}

The cellular response to injury is complex, and data from several laboratories have shed light on a cooperative interaction between TLRs and NLRs. In monocytes, macrophages, and several different cell lines, TLR2 has been shown to be essential for the NLRP3 inflammasome response to pathogens by assuring sufficient levels of NLRP3 and IL-1 $\beta$ messaging $[2,6]$. NLRP3 inflammasome activation requires not only the presence of NLRP3 increase in PI/annexin V staining detected by FACS analysis of primary RTE cells treated with ATP or nigericin compared to an untreated control. The error bars represent the standard deviations of 3 replicates, and the graph represents 1 of 3 similar experiments. d HMGB1 release from ligand-stimulated primary RTE cells. Lysates from WT versus NLRP3-/- RTE cells unstimulated (-) or stimulated with Pam3 or ATP were probed for HMGB1. The percent increase in optical density (OD) is noted in the graph. The data are representative of 4 identical experiments. but also a second signal, which then leads to activation of caspase-induced cell death and secretion of pyrogens such as IL-1 $\beta$ [2]. We have previously shown that blockade of TLR2 prevents renal tubular necrosis in a murine model of ischemia/reperfusion injury $[1,5]$. This study now examines whether TLR2 regulates the expression of NLRP3 and IL-1 $\beta$ in primary RTE cells and whether signaling through NLRP3 induces RTE cell death.

Primary RTEs from WT mice that were stimulated with the synthetic TLR2 ligand PAM3 increased their expression of NLRP3 mRNA (fig. 1a; PAM3). A ligand specific for the second signal of inflammasome activation, i.e. ATP, on the other hand, did not induce NLRP3 mRNA expression (fig. 1a; ATP) as expected. Stimulation of primary RTE cells with the TLR2 ligand PAM3 also induced 
IL-1 $\beta$ mRNA expression, whereas ATP had no effect (fig. $1 \mathrm{~b}$; PAM3 and ATP, respectively).

The role of NLRP3 inflammasome-mediated RTE cell death was examined in primary RTE cells harvested from either WT or NLRP3-/- mice. The RTE cells were treated with the NLRP3 ligand ATP or nigericin (a positive control for NLRP3 inflammasome activation). In figure 1c, it can be seen that treatment of the primary RTE cells with ATP significantly increased necrosis, as detected by the expression of PI and annexin (ATP) and the absence of NLRP3 (NLRP3-/-) blocked RTE cell death. Likewise, stimulation of NLRP 3 by nigericin increased cell death in primary RTE cells from WT mice but not in primary RTE cells from NLRP3-/- mice.

The induction of cell death in primary RTE cells via signaling through the NLRP3 inflammasome is further confirmed in figure 1d. RTE cells from either WT or NLRP3-/- mice were stimulated with PAM3 or ATP and cytoplasmic lysates probed for HMGB1. As noted in figure 1d, HMBG1 was detected in the lysates of ATP-stimulated WT RTE cells but not in the lysates of PAM3-stimulated RTE cells (WT). The release of HMGB1 from NLRP3-/- RTE cells treated with ATP, however, was significantly diminished compared to WT RTE cells. PAM3 had no effect on HMGB1 release in NLRP3-/- RTE cells (NLRP3-/-).

\section{Discussion}

The NLRP3 inflammasome is a multiprotein complex that is activated by a wide array of well-characterized stress signals, including ATP. Activation of the inflammasome accelerates caspase- 1 activation, resulting in the catalytic activation of pyrogens such as IL- $1 \beta$, and also caspase-1-mediated cell death (pyroptosis). During a stress response, two signals have been shown to be required for inflammasome complex formation and subsequent caspase- 1 activation [7]. The first signal provides adequate expression of inflammasome components (e.g. NLRP3) and its substrates (e.g. pro-IL-1 $\beta$ ), while a second signal is needed for inflammasome complex assembly and subsequent caspase- 1 activation. We have previously shown that NLRP3 is constitutively expressed in both human and rodent RTE cells [5]. Our data now show that NLRP 3 and IL- $1 \beta$ can be upregulated by TLR2 in RTE cells, and that stimulation through NLRP3 can directly induce death in primary RTE cells.

We have previously shown that necrosis and apoptosis of RTE cells can be inhibited in vivo by blockade of TLR2
[1] and NLRP3 [5], an observation confirmed by others $[8,9]$. TLR2 and NLRP3 have been shown to be upregulated in various different models of cellular injury [1013]. TLR2-induced NLRP3 upregulation occurs in monocytes, macrophages, and dendritic cells [2], but it is not known whether NLRP3 and IL- $1 \beta$ are upregulated in RTE cells under the influence of TLR2 signaling. Our study is the first, to our knowledge, to demonstrate TLR2-dependent upregulation of NLRP3 and IL- $1 \beta$ in primary murine RTE cells.

Our data also show that stimulation of the NLRP3 inflammasome by ATP causes necrosis of primary RTE cells. Presumably, ATP provides the second signal needed for inflammasome activation and caspase-1-mediated pyroptosis in the RTE cells in our model. In vivo models of renal ischemia/reperfusion injury have shown that the absence of NLRP3 can prevent both necrosis and apoptosis in murine kidneys [5]. Increasingly, it is being realized that several different renal diseases and systemic diseases affecting the kidney appear to involve the NLRP3 inflammasome [14], although whether NLRP3 and inflammasome signaling is regulated by TLR signaling has not been demonstrated in specific cell types within the kidney.

Interestingly, recent data have shown that TLRs and NLRs can simultaneously recognize virulence factors and microbial ligands, suggesting that TLRs and NLRs might be able to be activated at the same time [15]. Clearly, the complexity of TLR/NLR interaction is gaining appreciation. Future studies in our laboratory will focus on clarifying the complex interaction between TLR2 and NLRP3 in RTE cells and on the identification of mechanisms of inflammasome-mediated RTE cell death. Prevention of RTE cell death by blockade of either TLR2- or NLRP3mediated cell death mechanisms presents an emerging attractive therapeutic target for a variety of renal diseases that involve RTE cell death.

\section{Acknowledgment}

The work was supported by the following grants: NIH UABUCSD O'Brien Center grant P30 DK079337; NIH DK075718 (D.B.M.); NIH DK091136 (D.B.M.). 


\section{References}

$>1$ Shigeoka AA, et al: TLR2 is constitutively expressed within the kidney and participates in ischemic renal injury through both MyD88dependent and -independent pathways. J Immunol 2007;178:6252-6258.

-2 Segovia J, et al: TLR2/MyD88/NF-kappaB pathway, reactive oxygen species, potassium efflux activates NLRP3/ASC inflammasome during respiratory syncytial virus infection. PLoS One 2012;7:e29695.

3 Zager RA, Burkhart KM: Differential effects of glutathione and cysteine on $\mathrm{Fe}^{2+}, \mathrm{Fe}^{3+}$, $\mathrm{H}_{2} \mathrm{O}_{2}$ and myoglobin-induced proximal tubular cell attack. Kidney Int 1998;53:16611672.

4 Shigeoka AA, et al: Nod1 and nod2 are expressed in human and murine renal tubular epithelial cells and participate in renal ischemia reperfusion injury. J Immunol 2010;184: 2297-2304.
5 Shigeoka AA, et al: An inflammasome-independent role for epithelial-expressed Nlrp3 in renal ischemia-reperfusion injury. J Immunol 2010;185:6277-6285.

-6 Snodgrass RG, Huang S, Choi IW, Rutledge JC, Hwang DH: Inflammasome-mediated secretion of IL-1beta in human monocytes through TLR2 activation; modulation by dietary fatty acids. J Immunol 2013;191:43374347.

7 Bauernfeind FG, et al: Cutting edge: NF-kap$\mathrm{paB}$ activating pattern recognition and cytokine receptors license NLRP3 inflammasome activation by regulating NLRP3 expression. J Immunol 2009;183:787-791.

8 Iyer SS, et al: Necrotic cells trigger a sterile inflammatory response through the Nlrp3 inflammasome. Proc Natl Acad Sci USA 2009; 106:20388-20393.

-9 Leemans JC, et al; Renal-associated TLR2 mediates ischemia/reperfusion injury in the kidney. J Clin Invest 2005;115:2894-2903.

10 Tian J, et al: Extracellular HSP60 induces inflammation through activating and up-regulating TLRs in cardiomyocytes. Cardiovasc Res 2013;98:391-401.
11 Zhang J, et al: TLR2 mRNA upregulation in ischemic lobes in mouse partial hepatic ischemia/reperfusion injury model. J Huazhong Univ Sci Technolog Med Sci 2004;24:144146

12 Wolfs TG, et al: In vivo expression of Toll-like receptor 2 and 4 by renal epithelial cells: IFNgamma and TNF-alpha mediated up-regulation during inflammation. J Immunol 2002; 168:1286-1293.

13 Sandanger O, et al: The NLRP3 inflammasome is up-regulated in cardiac fibroblasts and mediates myocardial ischaemia-reperfusion injury. Cardiovasc Res 2013;99:164-174.

-14 Turner CM, Arulkumaran N, Singer M, Unwin RJ, Tam FW: Is the inflammasome a potential therapeutic target in renal disease? BMC Nephrol 2014;15:21.

15 Lin KM, et al: IRAK-1 bypasses priming and directly links TLRs to rapid NLRP3 inflammasome activation. Proc Natl Acad Sci USA 2014;111:775-780. 